\title{
Localized Upregulation of a New Expansin Gene Predicts the Site of Leaf Formation in the Tomato Meristem
}

\author{
Didier Reinhardt, Franz Wittwer, Therese Mandel, and Cris Kuhlemeier ${ }^{1}$ \\ Institute of Plant Physiology, University of Berne, Altenbergrain 21, CH-3013 Berne, Switzerland
}

\begin{abstract}
Expansins are extracellular proteins that increase plant cell wall extensibility in vitro and are thought to be involved in cell expansion. We showed in a previous study that administration of an exogenous expansin protein can trigger the initiation of leaflike structures on the shoot apical meristem of tomato. Here, we studied the expression patterns of two tomato expansin genes, LeExp2 and LeExp18. LeExp2 is preferentially expressed in expanding tissues, whereas LeExp18 is expressed preferentially in tissues with meristematic activity. In situ hybridization experiments showed that LeExp18 expression is elevated in a group of cells, called $I_{1}$, which is the site of incipient leaf primordium initiation. Thus, LeExp18 expression is a molecular marker for leaf initiation, predicting the site of primordium formation at a time before histological changes can be detected. We propose a model for the regulation of phyllotaxis that postulates a crucial role for expansin in leaf primordium initiation.
\end{abstract}

\section{INTRODUCTION}

In tomato, leaves are positioned in a spiral phyllotaxis, with a divergence angle of $\sim 135^{\circ}$. This highly regular pattern originates from the apex of the plant, where leaf primordia are initiated at the peripheral zone of the shoot apical meristem (reviewed in Steeves and Sussex, 1989; Lyndon, 1990; J ean, 1994). At predictable sites on the meristem, groups of cells become engaged in the formation of a bulge that undergoes morphogenesis to become a leaf (Poethig, 1997). All three layers that have been described in the shoot apical meristem, that is, the epidermal $(L 1)$, the subepidermal $(L 2)$, and the corpus (L3) layers, contribute to the leaf (Szymkowiak and Sussex, 1996).

What are the cellular events that induce the local bulging of meristem tissue? Primordium initiation could be driven by a local increase in the rate of cell division or by a reorientation of the plane of cell division, followed by expansion and differentiation processes in the proliferating tissue (Meyerowitz, 1997). Alternatively, bulging could be driven by local expansion of primordium initials, followed by division of the expanded cells.

Several lines of evidence suggest that local growth processes in plants can be initiated by cell expansion, with cell division being a secondary event (reviewed in Jacobs, 1997). There is also evidence concerning the importance of cell expansion in the earliest steps of primordium formation. First, when cell division in wheat seedlings was inhibited by $\gamma$ irradiation, primordium initiation continued through local cell expansion (Foard, 1971). Second, transformed tobacco

\footnotetext{
${ }^{1}$ To whom correspondence should be addressed. E-mail cris.
} kuhlemeier@pfp.unibe.ch; fax 41-31-332-20-59. plants with downregulated cell cycle activity had smaller meristems with fewer cells than the controls had, as was expected, but they formed leaves of almost normal shape and size, and at normal rates. In the mature leaves, decreased cell number was compensated by increased cell size (Hemerly et al., 1995). From these experiments, we concluded that regulation of cell expansion rather than cell division determines the rate of primordium initiation and the final shape and size of the plant and its organs.

Although the role of hormones in the regulation of cell expansion is well documented (Kende and Zeevaart, 1997), the downstream events that drive and control the actual expansion process are not understood in detail (reviewed in Cosgrove, 1997). Recently, a cell wall protein that exhibits in vitro wall expansion activity was identified and purified (McQueen-Mason et al., 1992), and it has been designated "expansin." Because no hydrolytic activity could be attributed to expansin, it is thought that expansins act by disrupting hydrogen bonds between microfibrils and the surrounding matrix in the cell wall, thus allowing them to slip (McQueen-Mason and Cosgrove, 1994). In a previous study, we tested the hypothesis that changes in the wall extensibility of meristem cells can initiate primordium formation. Using purified expansin, we showed that the exogenous application of this protein can induce local growth on the meristem, resulting in the formation of bulges that resemble early primordia (Fleming et al., 1997). In some cases, these bulges grew out to leaflike structures with several characteristics of young leaves, such as formation of trichomes, expression of a leaf marker gene, dorsoventrality, and the ability to influence the positioning of future primordia. Therefore, exogenously applied expansin is sufficient to mimic at 
least some aspects of the endogenous processes involved in leaf primordium initiation.

Expansins are encoded by gene families with up to a dozen members (Cosgrove, 1997). Some of them are expressed in several organs and tissue types, whereas others show very tight specificity in their spatial and temporal expression patterns (Cho and Kende, 1997; Rose et al., 1997), suggesting that regulation of cell wall extensibility could be controlled at least in part by differential regulation of the different expansin genes. Although purified expansin protein from a given species can be active on cell walls from different plants, suggesting that specificity is low (McQueenMason et al., 1992), it is not known whether the different expansin genes found in a given species encode proteins with different "loosening activities" or with different specificities toward different components of the cell wall.

Here, we report the cloning of a tomato expansin CDNA, LeExp18. LeExp18 is highly expressed in meristematic tissues. The expression of LeExp18 in the shoot apical meristem correlates with areas of incipient leaf primordium formation and is thus the earliest positive molecular marker for leaf primordium initiation described to date. Considering the in vitro and in vivo activities of expansin, LeExp18 may play a crucial role in the temporal and spatial regulation of primordium initiation.

\section{RESULTS}

\section{Cloning of a Tomato Expansin cDNA and Genomic Analysis of Tomato Expansins}

Exogenously applied expansin protein can initiate primordium formation on the shoot apical meristem of tomato plants (Fleming et al., 1997). To study the function of endogenous expansin in primordium initiation, we decided to clone expansin cDNAs that are expressed in the apex of tomato plants and to study their regulation. Using degenerate primers matching conserved sequences in the coding region of the cucumber expansin 1 gene, we amplified a fragment of a putative tomato expansin by reverse transcription-polymerase chain reaction. The amplification product was used as a probe to isolate a full-length CDNA, named LeExp18. LeExp18 represents a new tomato expansin gene exhibiting all of the basic features of expansin, as described by Shcherban et al. (1995). The open reading frame of the LeExp18 cDNA codes for a polypeptide of 255 amino acids (Figure 1A). The predicted protein contains a putative signal sequence of 18 amino acids and all of the cysteines and tryptophans that are conserved between expansins (Figure $1 \mathrm{~A}$; Shcherban et al., 1995). LeExp18 is most closely related to the fruit-specific LeExp1 (Rose et al., 1997), with 79\% amino acid identity.

Genomic DNA gel blot analysis was performed to determine the number of expansin genes in the tomato genome.
Probes were derived from LeExp18 and from LeExp2, a partial cDNA isolated from leaf RNA (kindly provided by $\mathrm{S}$. McQueen-Mason, University of York, York, UK, who is working on a complete analysis of the expansin gene family in tomato). Probes derived from the $3^{\prime}$ noncoding regions of LeExp2 and LeExp18 detected single bands and occasional weaker bands (Figure 1B). Most likely, LeExp2 and LeExp18 are single-copy genes. The patterns of the bands detected with the two probes are different, confirming that both probes are gene specific.

A probe containing part of the coding region of LeExp18 was used to estimate the number of LeExp18-related genes in the tomato genome. After hybridization and washing at high stringency, we observed a banding pattern that was indistinguishable from the pattern seen with the LeExp18 3' probe (Figure 1B, third gel; cf. with second gel). After lowstringency hybridization with the LeExp18 coding region probe, we detected several additional bands in the different samples, none of which comigrated with LeExp2 bands (Figure 1B, fourth gel; cf. with first gel). Thus, LeExp18 and LeExp2 are sufficiently divergent even in the coding region to prevent cross-hybridization. We conclude that LeExp18 is a member of a small subfamily of expansins within a larger expansin family.

\section{LeExp2 and LeExp18 Are Differentially Regulated}

Expression levels of LeExp2 and LeExp18 mRNAs were determined by RNA gel blot analysis by using the probes derived from their $3^{\prime}$ noncoding regions. Samples were isolated from the root, stem, expanding leaves, leaf primordia $\left(P_{4}\right.$ to $\left.P_{6}\right)$, vegetative apex (including the three youngest leaf primordia, $P_{1}$ to $P_{3}$ ), and developing flowers at various stages. LeExp18 was detected primarily in apical tissues such as the vegetative apex, in developing flowers, in leaf primordia, and in the upper stem. Notably, LeExp18 expression was low in leaves and in the lower stem tissue (Figure 1C). In contrast, LeExp2 was expressed primarily in the stem, in maturing flowers, in expanding leaves, and in leaf primordia; however, LeExp2 was not expressed in the vegetative apex. No expression of either LeExp2 or LeExp18 could be detected in roots (Figure $1 \mathrm{C}$ ).

From the experiments described above, we conclude that LeExp2 expression coincides primarily with expansion (leaves, stems, and flowers), whereas LeExp18 expression is associated with meristematic activity (apex, leaf primordia, and developing flowers), although there is some overlapping expression of both expansins in leaf primordia, flowers, and the stem. In this context, it is interesting that all of these plant parts combine expansion and meristematic activity. The stem retains meristematic activity in the cambium, and the young leaves of tomato are known to retain meristematic features for prolonged times during leaflet formation (Hareven et al., 1996; Chen et al., 1997). 
A $\quad \operatorname{cssxp}$

LeExp1

LeExp1

MAFSYSPFSSLFLLPFFFVFTEADYGGWQSGHATFYGGG MWPSVLLHFSLILLTAEARI PGVFTAGAWOSAHATFYGGS MGIIIFILVLLFVDSCENIVEGRI PGVYSGGSWETAHATFXGGS $* * \ldots+\ldots * * * * * * *$.

--KWCLPGTIRV--TATNFCPPNFALPNNNGGWNPPLQHFDMAEPAFLQ 135 QW--CHPGSPSI FITATNFCPPNFALPNDNGGWCNPPRPHFDLAMPMELH 138 NWKWCLPGNPSI LI TATNFCPPNYALPNDNGGWCNPPRPHFDLAMPMFLK 14

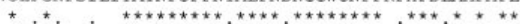

IAQYRAGIVPVSFRRVPCMKKGGVRFTINGHSYFNLVLITNVGGAGDVHS 185 IAEYRAGIVPVVYRRVPCRKOGGIRFTINGFRYFNLVLVTNVAGAGDIVS 18 LAQYRAGTVPYTYRRI PCRKOGGIRETTITGERYFNTVLITNVAGAGDIT 194 年

VSIKGSRTGWQSMSRNWGONWOSNNYINGOGISFOVTT GDGRTIMAYNIV

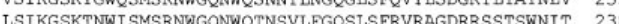
LSIKGSKTNWISHSRNGGQNWQTNSVLFGQSLSFRVRAGDRRSSTSWNIT 23 VWVKGTKTNWI PLSRNWGQNWQSNAVLTGQSLSFRVKASDHRSSTSWMMV 24

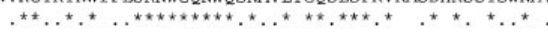

PSNWQFGQTYEGPQE-- 250 PAHWQFGQTFVGKNFRV 255 PSHWQFGQTFIGKNFKI 261

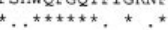

B

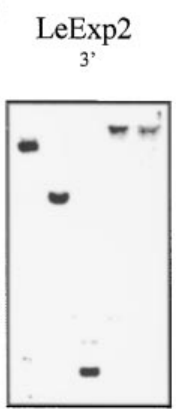

$\begin{array}{llllll}1 & 2 & 3 & 4 & 5\end{array}$

$$
\underset{3^{\prime}}{\operatorname{LeExp} 18}
$$

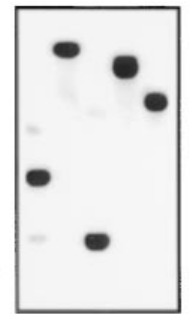

$\begin{array}{llllll}1 & 2 & 3 & 4 & 5\end{array}$

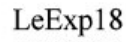
coding $\left(65^{\circ}\right)$

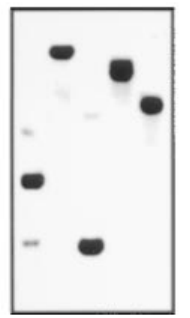

$\begin{array}{llllll}1 & 2 & 3 & 4 & 5\end{array}$
LeExp18 coding $\left(50^{\circ}\right)$

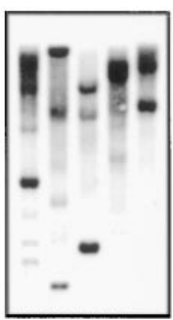

1223045
C

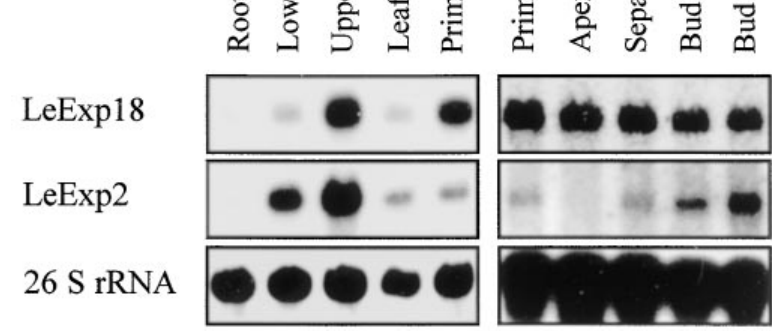

Figure 1. Characterization of LeExp18.

(A) Amino acid sequence alignment for cucumber expansin1 (CsExp1, previously designated CuExS1) with the tomato expansins isolated from the apex (LeExp18) and from fruits (LeExp1). Conserved positions with identical amino acids are marked with asterisks, and conservative substitutions are marked with dots. Dashes indicate gaps that were introduced to facilitate alignment. The numbering refers to the individual sequences.

(B) DNA gel blot analysis of tomato genomic DNA with probes from LeExp2 and LeExp18. DNA samples were digested with EcoRI
Both LeExp2 and LeExp18 were expressed in floral apices at various stages of development (Figure 1C). The increase in expression of LeExp2 with age could mean that also in floral apices, LeExp 2 expression is concentrated in expanding tissues.

\section{Expansin Genes Are Expressed in the Meristem}

To determine the cellular localization of expansin gene expression within the shoot apex, we performed in situ hybridizations. Figures $2 \mathrm{~A}$ to $2 \mathrm{H}$ show consecutive longitudinal sections through a vegetative apex. These sections were hybridized with a ${ }^{35} \mathrm{~S}$-labeled probe derived from the coding region of LeExp18. At left in Figures $2 \mathrm{~A}$ to $2 \mathrm{H}$, the youngest primordium $\left(\mathrm{P}_{1}\right)$ can be seen. The hybridization signal was distributed throughout the meristem and young primordia, and it extended into subepidermal tissues of the young stem (Figures $2 \mathrm{~A}$ to $2 \mathrm{H}$ ). Interestingly, the levels of expansin signal were elevated opposite to the youngest primordium $\left(P_{1}\right)$ (Figures $2 \mathrm{C}$ to $2 \mathrm{~F}$, arrowheads). Assuming that $\mathrm{P}_{2}$ is toward the observer and that the direction of the phyllotaxis is clockwise, this is the region in which the next primordium $\left(I_{1}\right)$ is expected to be initiated. However, from longitudinal sections, it is very difficult to establish the position of $\mathrm{I}_{1}$ with confidence.

In contrast to vegetative meristems, expression of LeExp18 in floral apices was much more diffuse, and no specific upregulation at the sites of organ initiation could be observed (Figures $2 \mathrm{I}$ to $2 \mathrm{~L}$ ).

\section{LeExp18 Is Specifically Upregulated at the Site of Incipient Primordium Initiation}

To determine the exact position of the local elevation in expansin $\mathrm{mRNA}$, we analyzed a series of transverse sections.

(lanes 1), EcoRV (lanes 2), HindIII (lanes 3), Xbal (lanes 4), or BamHI (lanes 5). After electrophoresis and blotting, the DNA was probed with radiolabeled probes from LeExp2 and LeExp18, as indicated. LeExp2 3' and LeExp18 3' designate the gene-specific probes from the $3^{\prime}$ untranslated regions. LeExp18 coding designates the probe from the coding region. Hybridization with this probe was performed under conditions of normal stringency $\left(65^{\circ} \mathrm{C}\right)$ or low stringency $\left(50^{\circ} \mathrm{C}\right)$.

(C) Expression of LeExp18 and LeExp2 in different tomato tissues. Total RNA $(5 \mu \mathrm{g})$ was isolated from roots, lower stems (downward from $3 \mathrm{~cm}$ below the apex) and upper stems $(0.2$ to $1.2 \mathrm{~mm}$ below the summit of the apex), leaves ( 3 to $5 \mathrm{~cm}$ in length), leaf primordia $\left(P_{4}\right.$ to $\left.P_{6}\right)$, vegetative apices (including the three youngest primordia $P_{1}$ to $P_{3}$ ), flowers at the stage of sepal initiation (sepal stage), and maturing flower buds of different lengths ( 1 or $10 \mathrm{~mm}$ ). The blots were analyzed with probes specific for LeExp18, LeExp2, or the $26 \mathrm{~S}$ subunit of rRNA. 

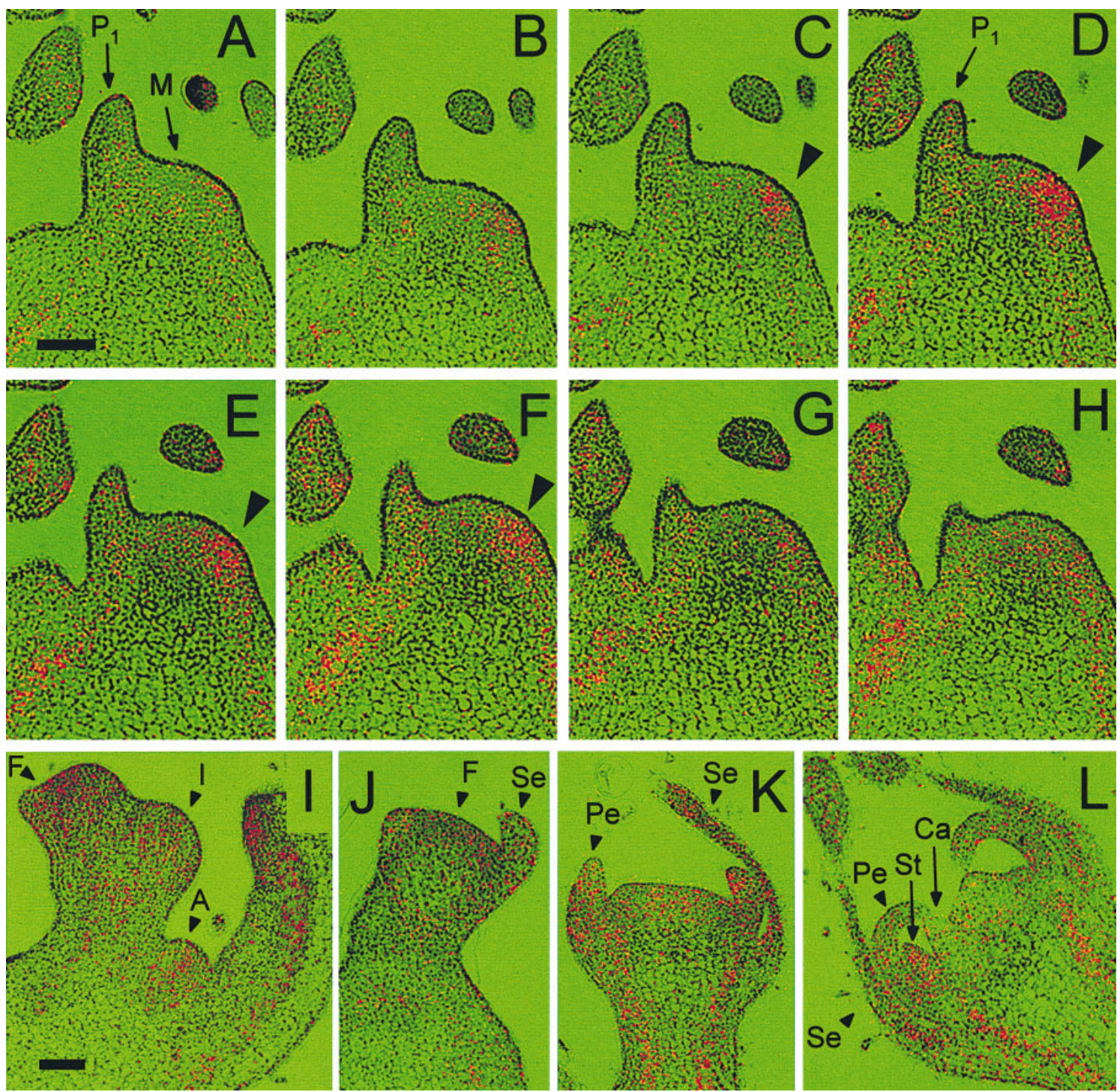

Figure 2. Expression of Expansin in the Vegetative Apex and in Developing Flowers of Tomato.

(A) to $\mathbf{( H )}$ Consecutive longitudinal sections $(7 \mu \mathrm{m})$ of a vegetative apex were analyzed by in situ hybridization. The sections were hybridized with a probe derived from the coding region of LeExp18. The sections exhibit an apex with the meristem dome in the center (M in [A]) and the youngest primordium (indicated with $P_{1}$ in $[\mathbf{A}]$ and $[\mathbf{D}]$ ) to the left. The site of increased signal levels is indicated by arrowheads in (C) to (F). Bar in (A) $=$ $100 \mu \mathrm{m}$.

(I) to (L) Longitudinal sections of tomato flowers at different stages of development were analyzed by in situ hybridization, using the same probe as given in (A) to (H). A, axillary meristem; Ca, carpel primordium; F, floral meristem; I, inflorescence meristem; Pe, petal primordium; Se, sepal primordium; St, stamen primordium. Bar in (I) $=100 \mu \mathrm{m}$.

Figures $3 \mathrm{~A}$ to $3 \mathrm{P}$ show a series of consecutive cross-sections of a vegetative tomato apex that were hybridized with a ${ }^{35} \mathrm{~S}$-labeled probe derived from the LeExp18 3' end. The oldest primordium $\left(\mathrm{P}_{3}\right)$ is in the lower half of the images (indicated in Figures $3 \mathrm{~A}$ and $3 \mathrm{H})$. The younger primordia $\left(\mathrm{P}_{2}\right.$ and $P_{1}$ ) follow in a counterclockwise, spiral phyllotaxis, with a divergence angle of $\sim 135^{\circ}$, so that the site of incipient primordium initiation $\left(I_{1}\right)$ can be predicted (indicated by arrowheads in Figures 3D to $3 \mathrm{~J})$. In the sections from the top of the apex (Figures $3 \mathrm{~A}$ to $3 \mathrm{C}$ ), LeExp18 was only expressed 
at low levels. Low levels of expansin signal could also be seen throughout the meristem, with a concentration of signal localized at $\mathrm{I}_{1}$. The elevated level is present in seven consecutive sections (Figures $3 \mathrm{D}$ to $3 \mathrm{~J}$ ). Considering that the thickness of the sections is $7 \mu \mathrm{m}$, we can estimate that the region of elevated expansin expression corresponds to an approximately circular area on the flank of the meristem with a diameter of $\sim 50 \mu \mathrm{m}$.

The tissue with elevated expansin expression comprises all three meristem layers (cf. Figures $2 \mathrm{D}$ and $3 \mathrm{H}$ ) and extends almost to the center of the meristem proper. Apart from the meristem, LeExp18 was also expressed in primor$\mathrm{dia}$ and in the cortical tissues of the young stem (Figures $3 \mathrm{~L}$ to $3 \mathrm{P})$. The expression pattern in primordia comprised a $\mathrm{U}$-shaped area that included the central tissues forming the vasculature and the adaxial margins of the leaf primordia, where leaflets and the leaf blade develop. The ring-shaped region of expansin expression in the stem coincides with developing vascular bundles and may reflect procambium activity. Therefore, high expression of LeExp18 coincides with tissues showing meristematic activity, such as the shoot apical meristem, the leaf margins, and the procambium, whereas expanding tissues, such as the central pith tissue in the stem and the adaxial and abaxial side of the rachis, exhibit low levels of LeExp18 expression. This is in contrast to the idea that expansin expression is associated with cell elongation in tissues such as leaves, hypocotyls, and grass internodes (Cho and Kende, 1997; Cosgrove, 1997). LeExp18 may therefore fulfill a specific function in meristematic tissues, particularly in leaf primordium initiation.

\section{Elevated Levels of LeExp18 Expression at $I_{1}$ Do Not Coincide with Markers for Cell Division and General Cell Metabolism}

Our previous results indicate that LeExp18 is expressed in meristematic areas and upregulated at the site of incipient primordium formation on the shoot apical meristem. To determine how specific this expression pattern is, we compared LeExp18 expression with the expression of marker genes for cell division and general cell metabolism.

In a dividing cell population, such as an actively growing meristem, cell growth occurs mainly during the $G_{1}$ phase. Cells enter the $\mathrm{S}$ phase only briefly before mitosis to replicate the genome (Fosket, 1994). During the S phase, genes for chromosomal proteins, such as the histones, are transcriptionally upregulated. Therefore, histone gene expression can be used as a marker for cell division activity in the meristem (Fleming et al., 1993; Brandstädter et al., 1994). We analyzed the levels of histone $\mathrm{H} 4 \mathrm{mRNA}$ in series of consecutive transverse sections. Figures $4 A$ to $4 D$ show four consecutive cross-sections that correspond to the sections shown in Figures $3 \mathrm{E}$ to $3 \mathrm{H}$, that is, Figure $4 \mathrm{~A}$ is the fifth section from the top. The four youngest primordia and the position of incipient primordium formation $\left(I_{1}\right.$, indicated by arrowheads) are indicated in Figures $4 \mathrm{~A}$ and 4D. In general, the distribution of histone $\mathrm{H} 4$ signal was consistent with histone expression patterns described earlier (Fleming et al., 1993; Brandstädter et al., 1994) and correlated with the most actively proliferating regions of the apex (Steeves and Sussex, 1989). Within the meristem, the signal was low in the uppermost sections (data not shown), whereas it was high in the organogenic region of the meristem (Figures 4A to 4D). Although histone $\mathrm{H} 4$ was distributed in a patchy pattern, presumably representing clusters of cells in the $\mathrm{S}$ phase (Fleming et al., 1993; Brandstädter et al., 1994), this pattern was random. Notably, levels of $\mathrm{H} 4$ signal were not elevated at $I_{1}$ (Figures 4A to 4D; $I_{1}$ is indicated by arrowheads), indicating that cell division activity was not significally increased at this position. Therefore, the local elevation of LeExp18 expression at $\mathrm{I}_{1}$ does not coincide with increased cell division activity (cf. Figures $3 \mathrm{E}$ to $3 \mathrm{H}$ and Figures $4 \mathrm{~A}$ to $4 \mathrm{D}$ ).

A particular pattern of mRNA distribution observed by using in situ hybridization analysis may have been caused by specific differential regulation. However, uneven distribution can also be caused by differences in cell size or general metabolic activity (for a discussion, see Mandel et al., 1995). Thus, to determine whether a given mRNA pattern is the result of specific regulation, we must compare the signal to a constitutive control. Previously, we used staining with acridine orange as a marker for RNA distribution in sections of shoot apical meristems (Fleming et al., 1993). These experiments clearly demonstrated that a gradient of RNA signal intensity can be observed, with the highest signal being in the meristem proper. We argued that this gradient largely reflects differences in cell size, because in highly expanded cells, the signal per volume unit is "diluted" by the volume of the vacuoles (Mandel et al., 1995). The distribution of the mRNA for a ribosomal protein, $\mathrm{Rp} 2$, essentially reflected total RNA detected with acridine orange and was used previously as a reliable constitutive control for general metabolic activity (Fleming et al., 1993).

Figures $4 \mathrm{E}$ to $4 \mathrm{H}$ show a series of consecutive cross-sections of an apex probed with rpl2. The sections are comparable to the sections depicted in Figures $3 \mathrm{~F}$ to $3 \mathrm{I}$ and exhibit an apex with five primordia in clockwise phyllotaxis (Figure $4 \mathrm{H})$. The highest levels of rpl2 signal were detected throughout the meristem proper and the youngest primordia $\left(\mathrm{P}_{1}\right.$ and $\left.P_{2}\right)$ and in the margins of older primordia $\left(P_{4}\right.$ and $\left.P_{5}\right)$. At lower levels, rpl2 was also expressed in the developing vasculature of primordia. The same pattern was found in experiments in which total RNA in consecutive transverse sections was stained with acridine orange (data not shown). Analysis of rpl2 expression in the apex demonstrates that general metabolic activity in the meristem is evenly distributed and is increased only in developing primordia (e.g., $\mathrm{P}_{2}$ in Figure $4 \mathrm{H}$ ). In contrast, LeExp18 expression is increased at $\mathrm{I}_{1}$ but is relatively low in young primordia (cf. Figures $4 \mathrm{H}$ and $3 \mathrm{H}$ ). This shows that the local elevation of LeExp18 expression does not reflect increased metabolic activity but is the result of specific upregulation at the site of incipient 

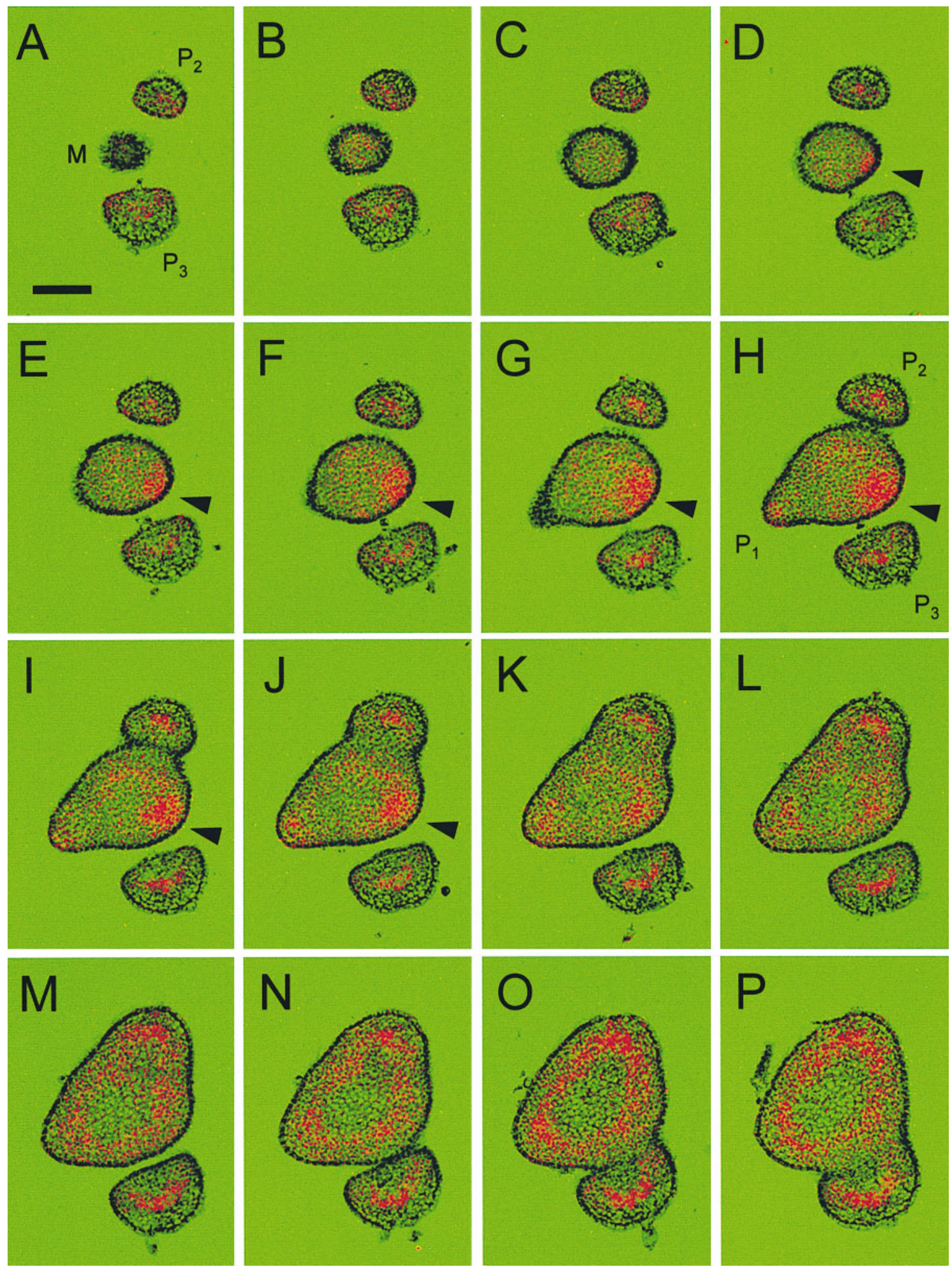

Figure 3. Specific Upregulation of LeExp18 Expression at the Site of Incipient Primordium Formation $\left(I_{1}\right)$.

Consecutive transverse sections $(7 \mu \mathrm{m})$ of a tomato apex were analyzed by in situ hybridization. The sections were hybridized with a probe specific for LeExp18. (A) is the top section; $(\mathbf{P})$ is the bottom section. Bar in (A) $=100 \mu \mathrm{m}$. 
primordium formation. Therefore, we postulate that the pattern of LeExp18 expression reflects the regulation of phyllotaxis in the meristem and that LeExp18 serves a specific function in leaf primordium initiation.

\section{DISCUSSION}

\section{Differential Expression of Expansin Genes}

We have isolated a tomato expansin cDNA, LeExp18, that represents a member of a small multigene subfamily of the tomato expansin genes. The tomato expansin family also includes LeExp2, an expansin cDNA isolated from leaves, and the fruit-specific LeExp1 cDNA, which may play a role during fruit softening (Rose et al., 1997). The existence of multiple expansin genes could signify that the individual proteins have distinct biochemical properties. Currently, however, neither sequence comparisons nor the limited functional data available indicate that expansins have distinct biochemical specificities; therefore, the major significance of the presence of multiple genes may be that distinctly expressed genes can regulate cell expansion in place and time.

The RNA gel blots shown in Figure $1 \mathrm{C}$ demonstrate that the LeExp2 and LeExp18 genes are differentially expressed. LeExp2 is not expressed in the shoot apical meristem but is predominantly active in more mature tissues. In flowers, LeExp2 expression increases during maturation. In contrast, LeExp18 expression is highest in the shoot apex and in flowers, with low expression in maturing leaves. Our research interest centers on the vegetative shoot apical meristem; therefore, we analyzed the expression of LeExp18 in this organ. Expansin gene expression was compared with the expression pattern of the housekeeping genes encoding ribosomal protein $\mathrm{Rp} / 2$ and histone $\mathrm{H} 4$. A comparison with housekeeping genes is essential, because differences in cell size and general metabolic activity can cause large apparent deviations from a uniform signal intensity (for a detailed discussion, see Mandel et al., 1995).

As can be seen from the hybridization patterns obtained with rpl2 (Figures $4 \mathrm{E}$ to $4 \mathrm{H}$ ), the small cells present in the shoot apical meristem, the young leaf primordia, and the meristematic zones at the margins of older primordia display the highest signal density. In contrast, the larger cells of the midrib and the stem pith tissue, for instance, have fewer sil- ver grains per square micrometer. The distribution of the hybridization signal coincides with the staining pattern seen with acridine orange, which is a general dye for nucleic acids (data not shown). Thus, the signal distribution seen with rpl2 reflects the general RNA distribution in the shoot apex and serves as a useful baseline against which the expression of other genes can be compared. Histone $\mathrm{H} 4$ is expressed in patches of cells throughout the apex, presumably reflecting those cells that are in $\mathrm{S}$ phase and actively dividing. It can also be seen that the areas of high cell division activity are the same areas that have high rpl2 expression. Thus, rpl2 is a general marker for small cells with high metabolic activity, whereas histone $\mathrm{H} 4$ expression defines the subset of the rpl2-expressing cells that are in $\mathrm{S}$ phase.

In the apex, the expression patterns of LeExp18 observed in in situ experiments exhibited two main features. First, LeExp18 expression in general coincided with small, rapidly dividing cells in the meristem, in leaf primordia, and in the vascular tissues of the young stem. Second, LeExp18 expression was elevated at the site of incipient primordium formation $\left(I_{1}\right)$ when compared with other regions in the meristem. This distribution was surprising considering the postulated function of expansins in cell elongation and cell enlargement, as discussed below (Cosgrove, 1997). Expression in the floral meristems was more diffuse, and no specific upregulation at the site of incipient primordium formation could be observed (Figures $2 \mathrm{I}$ to $2 \mathrm{~L}$ ).

\section{Role of Expansin in Leaf Primordium Formation}

The action of expansin and the expression of expansin genes are commonly associated with cell expansion in rapidly growing tissues, for example, in the unidirectional elongation of hypocotyls (Cosgrove, 1997) and grass internodes (Cho and Kende, 1997) or in expanding leaves (Keller and Cosgrove, 1995). This association may well hold for LeExp2, which is highly expressed in stems, maturing leaves, and flowers but is absent from meristematic tissues. However, LeExp18-expressing cells are small and actively dividing, and in these cells, upregulation of expansin expression is accompanied by isodiametrical cell expansion and cell division.

The differences in expression pattern of LeExp18 and histone $\mathrm{H} 4$ invite a discussion of the relative importance of cell expansion versus cell division in primordium initiation.

Figure 3. (continued).

(A) to (C) Sections from the top of the apex, exhibiting the apical meristem dome ( $M$ in $[\mathbf{A}]$ ) and two primordia $\left(P_{3}\right.$ and $P_{2}$ in $\left.[\mathbf{A}]\right)$. The youngest primordium cannot be seen at this level.

(D) to $(\boldsymbol{U})$ Sections including the region of primordium initiation. The sections represent the meristem in the center and the three youngest primordia in counterclockwise phyllotaxis $\left(P_{3}, P_{2}\right.$, and $P_{1}$ in $\left.[H]\right)$, with $P_{1}$ being the youngest primordium. The site of incipient primordium formation is indicated by arrowheads in (D) to ( ) .

(K) to (P) Sections from the lower apex, representing young stem tissue. 

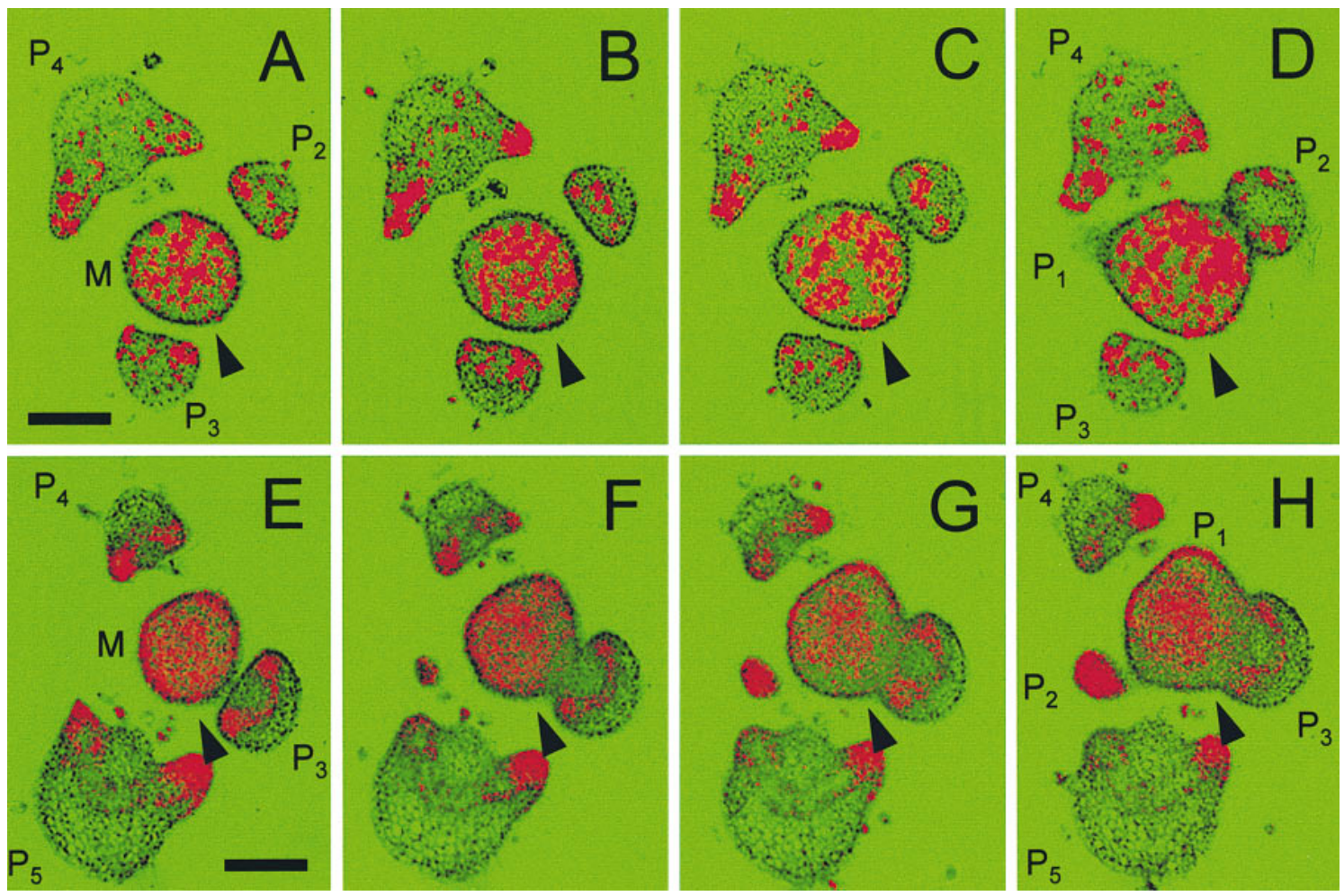

Figure 4. Expression in the Tomato Apex of Marker Genes for Cell Division and for General Metabolism.

(A) to (D) A series of consecutive transverse sections was analyzed by in situ hybridization. The sections were hybridized with a probe for the histone $\mathrm{H} 4$ transcript. The meristem is shown in the center (M in $[A])$, and primordia can be seen in counterclockwise phyllotaxis $\left(P_{4}, P_{3}, P_{2}\right.$, and $P_{1}$ in [A] and/or [D], with $P_{1}$ being the youngest). The site of incipient primordium formation is indicated by arrowheads. (A) is the fifth section from the top of the apex; (D) is the eighth section from the top. Bar in (A) $=100 \mu \mathrm{m}$.

(E) to $\mathbf{( H )}$ A series of consecutive transverse sections was analyzed by in situ hybridization. The sections were hybridized with the rpl2 probe. The meristem is shown in the center ( $M$ in $[E])$, and primordia can be seen in clockwise phyllotaxis $\left(P_{5}, P_{4}, P_{3}, P_{2}\right.$, and $P_{1}$ in $[E]$ and/or $[H]$, with $P_{1}$ being the youngest). The site of incipient primordium formation is indicated by arrowheads. $(E)$ is the sixth section from the top of the apex; $\mathbf{( H )}$ is the ninth section from the top. Bar in $\mathbf{( E )}=100 \mu \mathrm{m}$.

Whereas LeExp18 expression was clearly elevated in $I_{1}$ relative to the surrounding tissue, no obvious increase in histone $\mathrm{H} 4$ expression was observed. Although the difference between the two expression patterns was clear, we could not rule out subtle differences in expression level of histone $\mathrm{H} 4$ in various parts of the meristem. However, Lyndon (1968, 1970a, 1970b), in a careful study of division rates in vegetative pea apices, found that primordium initiation was not accompanied by significantly increased cell division rates. He concluded that primordium initiation is likely to be driven by changes in the orientation of cell division rather than by an increase in division rates. Recent work from several laboratories suggests, however, that plants are surprisingly tolerant of serious perturbations of both the rate and the orientation of cell division. Expression of a dominant negative form of the cell cycle regulator $\mathrm{cdc} 2$ led to a reduction of the rate of cell division without major effects on organogenesis (Hemerly et al., 1995). Similarly, the tangled mutation in maize, which interferes with the correct orientation of the cell plate, only affected the tissue anatomy in leaves without affecting the process of leaf formation or overall leaf shape (Smith et al., 1996). In our recent experiments, application of purified expansin protein to the $I_{2}$ position led to the formation of bulges and leaflike organs, indicating that a local elevation of expansin levels can induce organogenesis (Fleming et al., 1997). For these reasons, we favor a model in which leaf initiation is triggered by a localized upregulation of cell expansion. In this scenario, cell division is required primarily to subdivide the expanding tissue into smaller units but not to determine shape. In essence, both the rate and the orientation of cell division would be controlled by the localization of the tissue expansion.

In Figure 5, we propose a model for leaf initiation. In the first step, signaling from existing primordia, whether based on chemical gradients (Meinhardt, 1996), physical forces (Selker et al., 1992; Green et al., 1996), or both, specifies the 
$I_{1}$ region of the meristem (Figure $5 A$ ). This leads to differential activation or inactivation of regulatory genes in selected cells (Figure 5B, specification indicated by red triangles). Homeobox proteins of the Knotted class were shown in maize and Arabidopsis to be absent from the incipient primordia (Smith et al., 1992; Lincoln et al., 1994; Long et al., 1996). This expression pattern makes them excellent candidates to be controlled by a phyllotaxis-regulating mechanism. However, all Knotted-type genes described in tomato until now are ubiquitously expressed in the meristem (Hareven et al., 1996; Chen et al., 1997), and the comparable tomato gene(s) may as yet be undiscovered. Regional expression of regulatory genes could induce the regional expression of target genes, with LeExp18 prominent among them. Expression of LeExp18 will lead to bulging, which in turn activates a new genetic program and subsequent organogenesis (Figure $5 \mathrm{C}$ ).

We intend to use cell biological and genetic approaches to study the expression and the function of expansins in the
A

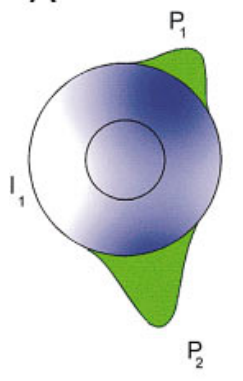

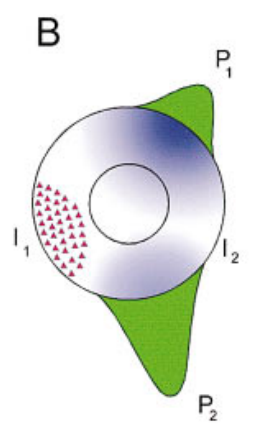

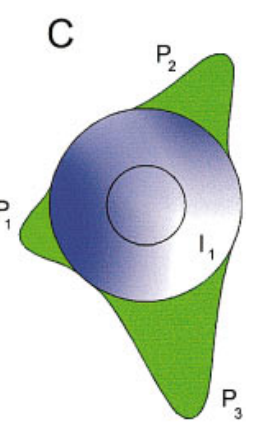

Figure 5. Model for the Regulation of Phyllotaxis in Tomato.

(A) An apex is represented showing the meristem (big circle) with the central zone (small circle) and the two youngest primordia $\left(\mathrm{P}_{2}\right.$ and $P_{1}$ ) in counterclockwise phyllotaxis. The area between the two circles represents the tissue from which the primordium initials are recruited (peripheral zone). The site of incipient primordium formation $\left(I_{1}\right)$ can be predicted from the positions of $P_{2}$ and $P_{1}$. According to the model, signals emanating from the existing primordia (blue gradients) determine the position of $I_{1}$. Due to unequal signaling from the existing primordia, the cells selected to initiate a primordium are closer to $\mathrm{P}_{2}$ than to $\mathrm{P}_{1}$.

(B) The selected cells at $I_{1}$ acquire a state of new identity represented by red triangles. This new identity is signified by upregulation of LeExp18. The LeExp18 protein increases tissue extensibility and will induce the tissue to form a bulge. At the same time, the influence of $P_{2}$ and $P_{1}$ decreases, as illustrated by the reduced range of the blue gradients. From the position of the LeExp18-expressing cells, the position of $\mathrm{I}_{2}$ can be deduced.

(C) The bulging tissue has undergone morphogenesis, and the resulting primordium is now called $\mathrm{P}_{1}$. This newly formed primordium is the source of signaling to subsequent primordium formation at $I_{1}$ (former $I_{2}$ ), whereas the influence of $P_{2}$ (former $P_{1}$ ) decreases further, and $P_{3}$ (former $P_{2}$ ) has almost no effect. The state in $(\mathbf{C})$ is equivalent to the state in $(\mathbf{A})$. expectation that this will shed light on the mechanisms underlying phyllotaxis.

\section{METHODS}

\section{Plant Growth}

Seeds of Lycopersicon esculentum cv Moneymaker were sown in soil and grown under defined conditions $\left(25^{\circ} \mathrm{C}\right.$ at $60 \%$ humidity, with $16 \mathrm{hr}$ of light and $8 \mathrm{hr}$ of dark) until the fifth to seventh plastochron stage was reached (Fleming et al., 1993). Apices were dissected and used for in situ hybridization or RNA extraction.

\section{Cloning of an Expansin CDNA}

Total RNA was isolated from tomato meristems (including $P_{1}$ ), as described by Fleming et al. (1996). After reverse transcription, polymerase chain reaction amplification was performed using two degenerate primers (sense primer 5'-ATGG GIGGIGCNTGYGGNTA-3' and antisense primer $5^{\prime}$-TGCCARTTYTGNCCCCARTT-3') to conserved sequences in the coding region of cucumber expansin1 (Fleming et al., 1997). The resulting band with the expected length of $\sim 500$ bp was used as a probe to screen a cDNA library derived from tomato shoots (kindly provided by A. Schaller, ETH Zürich, Switzerland). We isolated several positive clones, of which four were sequenced and confirmed to be identical to the probe used. One clone, LeExp18, was analyzed further and used to generate probes. The sequence of LeExp18 has GenBank accession number AJ 004997.

\section{Source of cDNAs Used for the Generation of Radiolabeled Probes}

For the generation of a nonspecific expansin probe, we subcloned a 156-bp fragment from the LeExp18 cDNA clone (nucleotides 194 to 350). As a gene-specific sequence from LeExp2, the $3^{\prime}$ untranslated region of the LeExp2 cDNA (kindly provided by S. McQueen-Mason, University of York, York, UK) containing nucleotides 659 to 906 (247 bp) was subcloned. The 3' probe from LeExp18 consisted of the 3' untranslated region of the LeExp18 CDNA spanning nucleotides 853 to 1130 (277 bp). Probes for these sequences were used in RNA and DNA gel blot analyses and in in situ hybridization experiments. The histone $\mathrm{H} 4 \mathrm{CDNA}$ was kindly provided by $\mathrm{K}$. Theres (University of Cologne, Cologne, Germany). The cDNA of rpl2 was described by Fleming et al. (1993).

\section{DNA Gel Blot Analysis}

High molecular weight total genomic DNA was isolated from young tomato plants, as described previously (Op den Camp and Kuhlemeier, 1997). DNA $(10 \mu \mathrm{g})$ was digested with EcoRI, EcoRV, HindIII, Xbal, or $\mathrm{BamHI}$, electrophoresed on $0.75 \%$ agarose gels, and transferred to Nytran N membranes (Schleicher \& Schuell). Blotting and hybridization procedures were performed under standard conditions (Sambrook et al., 1989). Blots were hybridized at $65^{\circ} \mathrm{C}$ ( $3^{\prime}$ probes) or $50^{\circ} \mathrm{C}$ (coding region probe), with randomly labeled probes made from the subcloned expansin cDNA fragments described above. The 
final wash was in $0.2 \times \mathrm{SSC}(1 \times \mathrm{SSC}$ is $0.15 \mathrm{M} \mathrm{NaCl}$ and $0.015 \mathrm{M}$ sodium citrate) and $0.1 \% \mathrm{SDS}$ at $65^{\circ} \mathrm{C}\left(3^{\prime}\right.$ probes) or $50^{\circ} \mathrm{C}$ (coding region probe).

\section{RNA Gel Blot Analysis}

RNA gel blot analysis was performed essentially as described by $O p$ den Camp and Kuhlemeier (1997). Total RNA from various parts of tomato plants was isolated as described by Fleming et al. (1993). To isolate $5 \mu \mathrm{g}$ of total RNA, we extracted 150 apices (including the three youngest primordia) and 450 primordia $\left(P_{4}\right.$ to $\left.P_{6}\right)$. RNA was quantified spectrophotometrically at $260 \mathrm{~nm}$, and $5-\mu \mathrm{g}$ portions were run on a $1.0 \%$ agarose-glyoxal gel after glyoxylation. RNA blotting and hybridization procedures were performed under standard conditions (Sambrook et al., 1989). Blots were hybridized at $65^{\circ} \mathrm{C}$, with randomly labeled probes from the expansin CDNAs described above or with a probe from a rRNA (Fleming et al., 1993). The final wash was in $0.1 \times \mathrm{SSC}$ and $0.1 \% \mathrm{SDS}$ at $65^{\circ} \mathrm{C}$.

\section{In Situ Hybridizations}

In situ hybridization experiments were performed according to the protocol described by Fleming et al. (1993), with minor modifications. From embedded tomato apices, we cut consecutive longitudinal or transverse sections $(7 \mu \mathrm{m})$ and used them for in situ hybridization. Before exposure, slides were treated with a solution of $50 \mu \mathrm{g} / \mathrm{mL}$ RNase A (rather than $1 \mu \mathrm{g} / \mathrm{mL}$ ) and finally washed in $2 \times$ SSC at room temperature and in $0.1 \times \mathrm{SSC}$ at $42^{\circ}$, each for $30 \mathrm{~min}$. After development, the slides were stained in toluidine blue and viewed on an LSM 310 microscope (Carl Zeiss AG, Oberkochen, Germany). Images were taken under bright-field light (shown in false green color) and overlayed with epifluorescence images taken under polarized light exhibiting the silver grain signal (shown in false red color). In all cases, control hybridizations were performed with the corresponding sense probes, and in all instances, the signal obtained was negligible compared with that obtained using the antisense probe.

\section{ACKNOWLEDGMENTS}

We thank Andreas Schaller for kindly providing a tomato shoot CDNA library, Simon McQueen-Mason for supplying a cDNA clone for LeExp2, Klaus Theres for a histone H4 CDNA, and Isabelle Dupuis, Andrew Fleming, and J eroen Stuurman for critical reading of the manuscript. This work was supported in part by a grant from the Swiss National Science Foundation.

Received February 23, 1998; accepted J uly 9, 1998.

\section{REFERENCES}

Brandstädter, J ., Rossbach, C., and Theres, K. (1994). The pattern of histone $\mathrm{H} 4$ expression in the tomato shoot apex changes during development. Planta 192, 69-74.
Chen, J .-J ., J anssen, B.-J ., Williams, A., and Sinha, N. (1997). A gene fusion at a homeobox locus: Alterations in leaf shape and implications for morphological evolution. Plant Cell 9, 1289-1304.

Cho, H.-T., and Kende, H. (1997). Expression of expansin genes is correlated with growth in deepwater rice. Plant Cell 9, 1661-1671.

Cosgrove, D.J . (1997). Relaxation in a high-stress environment: The molecular bases of extensible cell walls and cell enlargement. Plant Cell 9, 1031-1041.

Fleming, A.J ., Mandel, T., Roth, I., and Kuhlemeier, C. (1993). The patterns of gene expression in the tomato shoot apical meristem. Plant Cell 5, 297-309.

Fleming, A.J ., Manzara, T., Gruissem, W., and Kuhlemeier, C. (1996). Fluorescent imaging of GUS activity and RT-PCR analysis of gene expression in the shoot apical meristem. Plant J. 10, 745-754.

Fleming, A.J ., McQueen-Mason, S., Mandel, T., and Kuhlemeier, C. (1997). Induction of leaf primordia by the cell wall protein expansin. Science 276, 1415-1418.

Foard, D.E. (1971). The initial protrusion of a leaf primordium can form without concurrent periclinal cell divisions. Can. J. Bot. 49, 694-702.

Fosket, D.E. (1994). Plant Growth and Development. (San Diego, CA: Academic Press).

Green, P.B., Steele, C.S., and Rennich, S.C. (1996). Phyllotactic patterns: A biophysical mechanism for their origin. Ann. Bot. 77, 515-527.

Hareven, D., Gutfinger, T., Parnis, A., Eshed, Y., and Lifschitz, E. (1996). The making of a compound leaf: Genetic manipulation of leaf architecture in tomato. Cell 84, 735-744.

Hemerly, A., de Almeida-Engler, J ., Bergounioux, C., Van Montagu, M., Engler, G., Inzé, D., and Ferreira, P. (1995). Dominant negative mutants of the $\mathrm{Cdc} 2$ kinase uncouple cell division from iterative plant development. EMBO J . 14, 3925-3936.

J acobs, T. (1997). Why do plant cells divide? Plant Cell 9, 1021-1029.

J ean, R.V. (1994). Phyllotaxis: A Systematic Study in Plant Morphogenesis. (Cambridge, UK: Cambridge University Press).

Keller, E., and Cosgrove, D.J . (1995). Expansins in growing tomato leaves. Plant J . 8, 795-802.

Kende, H., and Zeevaart, J .A.D. (1997). The five "classical" plant hormones. Plant Cell 9, 1197-1210.

Lincoln, C., Long, J ., Yamaguchi, J ., Serikawa, K., and Hake, S. (1994). A knotted1-like homeobox gene in Arabidopsis is expressed in the vegetative meristem and dramatically alters leaf morphology when overexpressed in transgenic plants. Plant Cell 6, 1859-1876.

Long, J .A., Moan, E.I., Medford, J .I., and Barton, M.K. (1996). A member of the KNOTTED class of homeodomain proteins encoded by the STM gene of Arabidopsis. Nature 379, 66-69.

Lyndon, R.F. (1968). Changes in volume and cell number in the different regions of the shoot apex of Pisum during a single plastochron. Ann. Bot. 32, 371-390.

Lyndon, R.F. (1970a). Rates of cell division in the shoot apical meristem of Pisum. Ann. Bot. 34, 1-17.

Lyndon, R.F. (1970b). Planes of cell division and growth in the shoot apex of Pisum. Ann. Bot. 34, 19-28. 
Lyndon, R.F. (1990). Plant Development: The Cellular Basis. (London: Unwin Hyman).

Mandel, T., Fleming, A.J ., Krähenbühl, R., and Kuhlemeier, C. (1995). Definition of constitutive gene expression in plants: The translation initiation factor $4 \mathrm{~A}$ gene as a model. Plant Mol. Biol. 29, 995-1004.

McQueen-Mason, S.J ., and Cosgrove, D.J . (1994). Disruption of hydrogen bonding between plant cell wall polymers by proteins that induce wall extension. Proc. Natl. Acad. Sci. USA 91, 6574-6578.

McQueen-Mason, S.J., Durachko, D.M., and Cosgrove, D.J . (1992). Two endogenous proteins that induce cell wall extension in plants. Plant Cell 4, 1425-1433.

Meinhardt, H. (1996). Models of biological pattern formation: Common mechanism in plant and animal development. Int. J. Dev. Biol. 40, 123-134.

Meyerowitz, E.M. (1997). Genetic control of cell division patterns in developing plants. Cell 88, 299-308.

Op den Camp, R.G.L., and Kuhlemeier, C. (1997). Aldehyde dehydrogenase in tobacco pollen. Plant Mol. Biol. 35, 355-365.

Poethig, R.S. (1997). Leaf morphogenesis in flowering plants. Plant Cell 9, 1077-1087.

Rose, J.K.C, Lee, H.H., and Bennett, A.B. (1997). Expression of a divergent expansin gene is fruit-specific and ripening-regulated. Proc. Natl. Acad. Sci. USA 94, 5955-5960.
Sambrook, J., Fritsch, E.F., and Maniatis, T. (1989). Molecular Cloning: A Laboratory Manual, 2nd ed. (Cold Spring Harbor, NY: Cold Spring Harbor Laboratory Press).

Selker, J .M.L., Steucek, G.L., and Green, P.B. (1992). Biophysical mechanisms for morphogenetic progression at the shoot apex. Dev. Biol. 153, 29-43.

Shcherban, T.Y., Shi, J., Durachko, D.M., Guiltinan, M.J., McQueen-Mason, S.J., Shieh, M., and Cosgrove, D.J . (1995). Molecular cloning and sequence analysis of expansins-A highly conserved, multigene family of proteins that mediate cell wall extension in plants. Proc. Natl. Acad. Sci. USA 92, 9245-9249.

Smith, L.G., Greene, B., Veit, B., and Hake, S. (1992). A dominant mutation in the maize homeobox gene, knotted-1, causes its ectopic expression in leaf cells with altered fates. Development 116, 21-30.

Smith, L.G., Hake, S., and Sylvester, A.W. (1996). The tangled-1 mutation alters cell division orientations throughout maize leaf development without altering leaf shape. Development 122, 481-489.

Steeves, T.A., and Sussex, I.M. (1989). Patterns in Plant Development. (New York: Cambridge University Press).

Szymkowiak, E.J ., and Sussex, I.M. (1996). What chimeras can tell us about plant development. Annu. Rev. Plant Physiol. Plant Mol. Biol. 47, 351-376. 